\title{
Beobachtungen zum Auftreten beschädigter Varroamilben im natürlichen Totenfall bei Völkern von Apis mellifera carnica
}

\author{
R Moosbeckhofer \\ Höhere Bundeslehr- und Versuchsanstalt für Wein- und Obstbau mit Institut für Bienenkunde, \\ Institut für Bienenkunde, Hauptstraße 14, A-2540 Bad Vöslau, Österreich
}

(eingegangen 8 März 1992; angenommen 11 August 1992)

\begin{abstract}
Zusammenfassung - Der natürliche Varroa-Totenfall von 111 Völkern auf 4 Standorten wurde vom 19.8-18.9.9.1991 erfaßt und auf Panzer- und Beinbeschädigungen untersucht. Helle adulte Milben waren häufiger beschädigt, als dunkle Milben. Die Anzahl beschädigter Varroamilben zeigte eine signifikante positive Korrelation mit der Höhe des Totenfalles. Der prozentuelle Anteil beschädigter Milben zeigte eine signifikante negative Korrelation mit dem Bienen- und Brutbefall, sowie dem Varroa-Abfall nach einer Behandlung mit Apistan und Bayvarol. Dies deutet darauf hin, daß ein aktives Abwehrverhalten der europäischen Honigbiene auch die Populationsentwicklung der Varroamilbe beeinflußt. Der Anteil beschädigter Varroamilben im natürlichen Totenfall könnte somit ein wichtiges Auslesekriterium auf dem Weg zu einer varroatoleranten Carnica-Biene sein.
\end{abstract}

Apis mellifera carnica / Varroa-Resistenz / Amputation / Verteidigungsverhalten

\section{EINLEITUNG}

Der natürliche Varroatotenfall, wie er durch eine Bodeneinlage beobachtet werden kann, widerspiegelt die Populationsentwicklung und Vermehrungstätigkeit der Varroamilbe. Er enthält aber auch Informationen über eine aktive VarroaAbwehr der europäischen Honigbiene. Diese wurden jedoch lange Zeit nicht als solche erkannt oder falsch gedeutet. Für Apis cerana liegen bereits seit längerer Zeit Beweise für eine aktive VarroaAbwehr vor (Peng et al, 1987).

Der österreichische Berufsimker A Wallner (1989, 1990a,b; 1991; 1992) po- stulierte erstmals aufgrund seiner Beobachtungen, daß die an toten Varroamilben immer wieder zu beobachtenden Verletzungen des Panzers - ebenso wie teilweise oder komplette Beinamputationen Ausdruck eines aktiven Abwehrverhaltens der Bienen seien, und nicht auf die Einwirkung von Ameisen, Ohrwürmern oder Speckkäfern zurückzuführen sind, wie man bisher glaubte.

Mittlerweile wurden seine Beobachtungen auch von anderen Autoren bestätigt (Ruttner, 1991; Ruttner und Hänel, 1992). Damit ist gesichert, daß auch die europäische Honigbiene in der Lage ist, Varroamilben aktiv zu entfernen und die Milben dabei schwer zu beschädigen. 
In der vorliegenden Arbeit sollte untersucht werden, ob zwischen dem Prozentsatz beschädigter Varroamilben und dem Bienen- und Brutbefall ein Zusammenhang besteht. Zusätzlich sollten eventuell vorhandene Unterschiede im Anteil beschädigter Varroamilben zwischen Einzelvölkern bzw. Völkergruppen verschiedener Abstammung erfaßt werden.

Anhand des Varroa-Abfalls nach einer chemischen Behandlung sollte geklärt werden, ob sich Auswirkungen einer aktiven Varroa-Abwehr seitens der Bienen auf die Varroa-Gesamtpopulation eines Bienenvolkes feststellen lassen.

Der Prozentsatz beschädigter Varroamilben wurde ermittelt und mit dem Bienen- und Brutbefall, dem Standort, der Königinnen-Abstammung und dem Varroa-Abfall nach einer chemischen $\mathrm{Be}$ handlung in Beziehung gesetzt.

\section{MATERIAL UND METHODE}

Auf 4 Standorten wurde an 111 Bienenvölkern von Apis mellifera carnica zwischen dem August und dem September 1991 der natürliche Varroatotenfall mit Hilfe von gittergeschützten Bodeneinlagen erfaßt und in ca 10-tägigen Intervallen ausgezählt.

Die Varroamilben wurden in Unbeschädigte und Beschädigte eingeteilt. Die Beschädigungen bestanden aus ganz oder teilweise amputierten Beinen sowie Panzerverletzungen (Stücke aus dem Panzer fehlten). Bei manchen Milben waren sowohl Bein-, als auch Panzerverletzungen zu beobachten. Die Beurteilung der Beschädigungen erfolgte mit Hilfe eines Stereomikroskops.

Adulte "helle" (= unreife) und "dunkle" (= reife) Milben wurden dabei getrennt erfaßt, um zu klären, ob helle oder dunkle Milben häufiger beschädigt werden.

Die Abgrenzung heller und dunkler Milben erfolgte aufgrund eigener Erfahrungen und stimmt annähernd mit der Farbstufe "ockerbraun" der Farbkarte RAL 80001 überein. Diese
Farbstufe wird nach Untersuchungen in Oberursel (mündliche Mitteilung Prof Ruttner) als die hellste Farbstufe reifer Varroaweibchen angesehen.

Alle Völker waren in Magazinbeuten auf 2 Zargen im Zander-Flachzargenmaß (Rähmchen-Außenmaße: $42 \times 16 \mathrm{~cm}$ ) untergebracht. Tabelle I bringt eine Übersicht über Völkerzahl und Volksstärken an den verschiedenen Standorten.

Im Anschluß an die letzte Auswertung des natürichen Totenfalles erfolgte bei allen Völkern eine chemische Varroabehandlung mit Apistan bzw Bayvarol.

Zur Bestimmung des prozentuellen Bienenbefalls [= (Anzahl Varroa/Anzahl Bienen) $\times 100$ ] wurden durchschnittlich 304 (sd = 76) Bienen von zentralen Brutwaben in Tiefküilbeutel abgeschüttelt, mit Äther abgetötet, tiefgekühlt, auf einer elektronischen Waage mit Zählmodul gezählt und mit einem elektrischen Rüttelsieb (Type: Retsch-Vibro) unter dauerndem Wasserdurchfluß ausgewaschen. Vorhandene Varroamilben wurden in einem feinen Sieb aufgefangen und gezählt.

Der Brutbefall ( $=$ [Anzahl befallener Zellen/ Gesamtzahl untersuchter Zellen] x 100) wurde an tiefgekühlten Wabenstücken mit gedeckelter

Tabelle I. Völkerzahl und mittlere Volksstärke (Standardabweichung) an den untersuchten Standorten.

\begin{tabular}{lrcc}
\hline Ort & $n=$ & $\begin{array}{c}\text { belagerte } \\
\text { Waben } \\
(x \text { sd) }\end{array}$ & $\begin{array}{c}\text { Brutwaben } \\
x(s d)\end{array}$ \\
\hline & & & \\
Grinzing* & 28 & $12,8(3,3)$ & $4,2(1,9)$ \\
Gainfarn* $^{*}$ & 31 & $14,3(3,3)$ & $5,5(1,7)$ \\
Pötzleinsdorf* $^{*}$ & 28 & $13,3(1,9)$ & $2,4(1,2)$ \\
Miesenbach* & 4 & $12,2(2,6)$ & $4,0(0,8)$ \\
Miesenbach** & 20 & $18,7(1,6)$ & $4,6(1,2)$
\end{tabular}

- = unbehandelte Jungvölker, die zwischen Anfang und Ende Juli 1991 aus Kunstschwärmen aufgebaut wurden; ${ }^{* *}=$ Wirtschaftsvölker (1990 aus Kunstschwärmen aufgebaut. Varroabehandlung erst nach der Überwinterung im Frühjahr 1991 mit Apistan). 
Brut (überwiegend Puppen mit dunklen Augen) ermittelt. Pro Volk wurden durchschnittlich 200 Zellen $(s d=70$ ) einzeln geöffnet und auf ihren Varroabefall untersucht.

Die Entnahme der Brut- und Bienenproben erfolgte in der ersten Septemberwoche 1991 jeweils bei allen Völkern eines Standortes am gleichen Tag).

Die statistische Auswertung erfolgte mit dem Statistikpaket "Statgraphics".

\section{ERGEBNISSE}

Der natürliche Varroatotenfall zeigte sowohl mit dem Bienenbefall $(n=67, r=$ $0,60, p<0.00001, y=51,37+11,57 x)$ als auch mit dem Brutbefall $(n=65, r=0,64$, $p<0.00001, y=43+5,74 x)$ eine höchst signifikante positive Korrelation.

Die 4 Standorte zeigten keine signifikanten Unterschiede im Prozentsatz beschädigter Milben (gewertet wurden nur Völker mit mehr als 20 gefundenen Varroamilben). Abb 1 faßt die Werte aller 4 Standorte zusammen und gibt einen Überblick über die zahlenmäßige Verteilung der Völker auf 9 Beschädigungsklassen.

Von insgesamt 8452 gefundenen Milben ( $=$ Summe aller Völker) wiesen 1601 (= 18.9\%) Beschädigungen auf (Tabelle II).

Die hellen Milben waren häufiger beschädigt ( $\bar{x}=28,6 \%, s d=16,8, n=69)$, als die dunklen Milben ( $\bar{x}=9,9 \%, s d=8,3, n=$ 69). Dieser Unterschied ist statistisch abgesichert ( $p<0,0001, n=69)$.

Zwischen den Völkern bestehen signifikante, völkertypische Unterschiede im Prozentsatz beschädigter Varroamilben (In die Mittelwertberechnungen aus den 3 Beobachtungsintervallen gingen nur 17 Völker mit mehr als 20 toten Varroamilben pro Beobachtungsintervall ein; Gesamtmittelwert aus 51 Beobachtungen: $\bar{x}=17,1 \%, s d=$ 10,$7 ; p=0,03$, Minimalwert: Volk 196: $\bar{x}=$

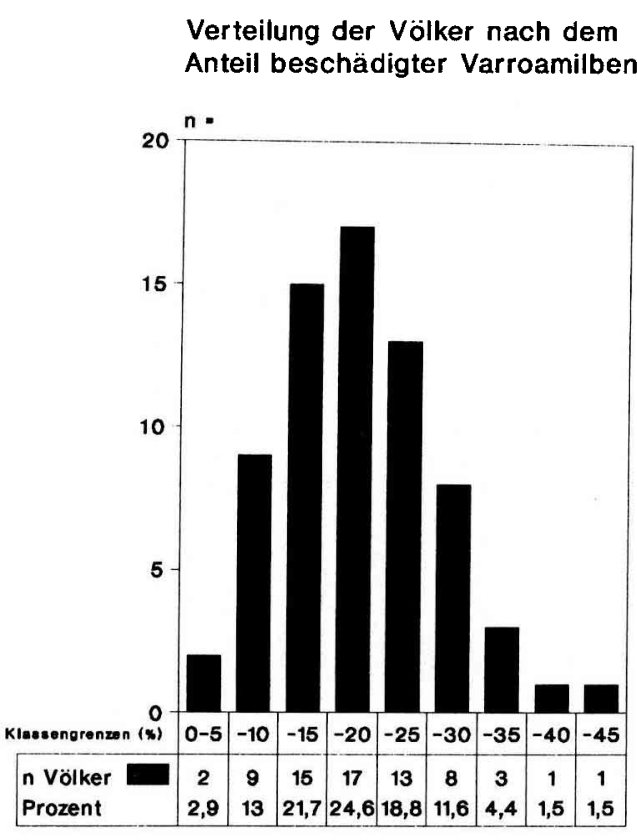

Abb 1. Verteilung der Völker nach Beschädigungsklassen (Nur Völker mit einem natürlichen Varroatotenfall von mehr als 20 Milben/Volk wurden berücksichtigt.

$9,0 \%, s d=7.6$; Maximalwert: Volk $79: \bar{x}=$ $36,3 \%, s d=15$ ).

Zwischen dem natürlichen Varroatotenfall und der Anzahl beschädigter Varroamilben zeigte sich eine höchst signifikante lineare Korrelation $(n=111, r=0,87, p<$ $0,0001, y=20,3+3,87 x$ ). Dieser Befund deckt sich mit den Beobachtungen von Ruttner und Hänel (1992).

Vier Gruppen von Königinnen unterschiedlicher Herkunft und Anpaarung zeigten keine signifikanten Unterschiede im Prozentsatz beschädigter Varroamilben.

Der Prozentsatz heller beschädigter Milben zeigte mit dem Brutbefall eine schwache, negative Korrelation. Mit dem Bienenbefall war keine signifikante Korrelation feststellbar. 


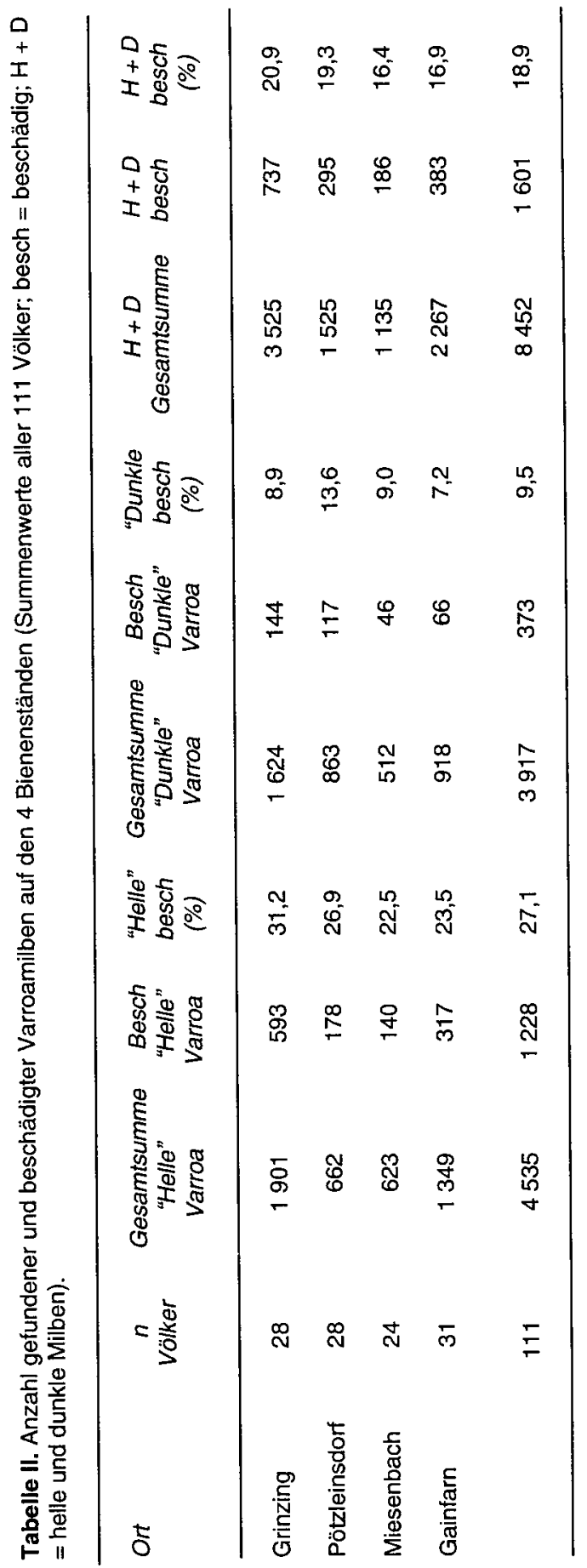


Der Prozentsatz beschädigter dunkler Milben zeigte sowohl mit dem Brut- als auch mit dem Bienenbefall (Abb 2, 3) eine höchstsignifikante negative Korrelation (reziprokes Modell: $1 / y=a+b x$, Korrelationen und Signifikanzwerte siehe Tabelle III).

Zwischen dem Varroa-Abfall nach einer chemischen Behandlung mit Apistan bzw. Bayvarol und dem Prozentsatz beschädigter Varroamilben wurde eine signifikante negative Korrelation gefunden (Abb $4 ; n=$ $7 ; r=-0,83 ; p=0,02 ; y=3980,21 \mathrm{X} \sim$ 1,012 [Multiplikatives Modell: $\left.y=a . X^{\wedge} b\right]$ ).

Durch die geringe Anzahl von 7 Völkern, die in die Berechnungen eingingen (natürlicher Varroatotenfall > 16), und dem nur schwachen Varroabefall von maximal 533 Milben in der auswertbaren Gruppe (= schwach befallene, ungestörte Wirtschafts-

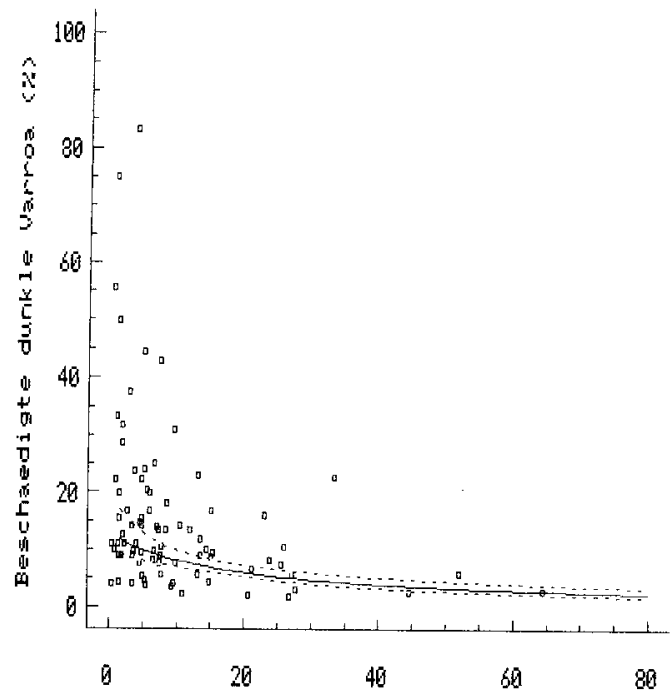

Brutbetall (\%)

Abb 2. Beziehung zwischen dem Prozentsatz beschädigter dunkler Varroamilben und dem Brutbefall (Innere strichlierte Linien: 95\% Vertrauensgrenzen; Äußere strichlierte Linien: 95\% Vorhersagegrenzen).

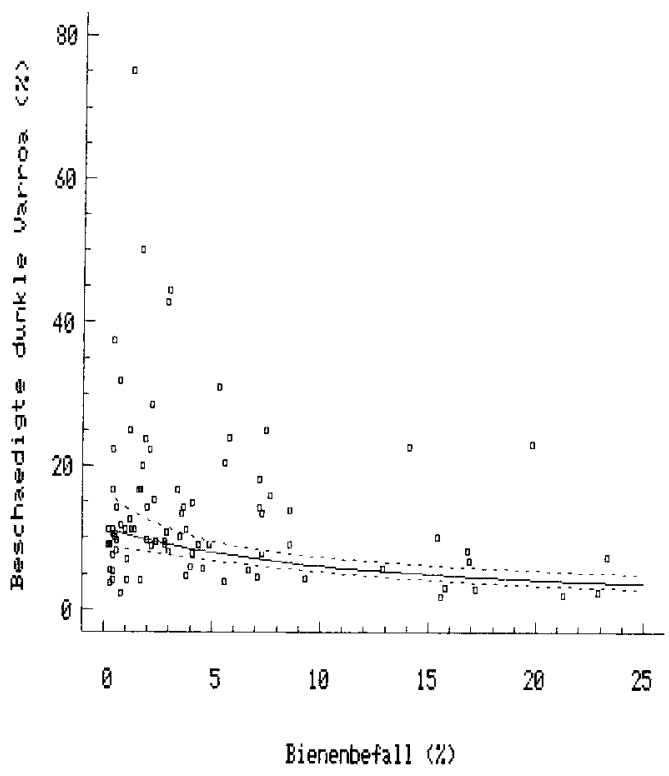

Abb 3. Beziehung zwischen dem Prozentsatz beschädigter dunkler Varroamilben und dem Bienenbefall.

völker aus Miesenbach) ist dieses Ergebnis jedoch nur mit großer Vorsicht zu betrachten.

\section{DISKUSSION}

Die negative Korrelation des Prozentsatzes beschädigter Varroamilben mit dem Brut- und Bienenbefall und dem Abfall nach einer chemischen Behandlung zeigt, daß dieses Merkmal ein mögliches Auslesekriterium bei der Zucht einer varroatoleranten Biene darstellt, sofern es zwischen einzelnen Völkern genetisch bedingte Unterschiede gibt.

Ob die in dieser Arbeit gefundenen beträchtlichen Unterschiede zwischen verschiedenen Völkern (zum Beispiel Volk 74: $4,5 \%$ beschädigte Varroamilben [Summe 
Tabelle III. Korrelationskoeffizienten, Signifikanzwerte und Regressionsgleichungen für die Beziehung zwischen hellen und dunklen beschädigten Varroamilben und dem Bienen- bzw. Brutbefall.

Bienenbefall

nicht

beschädigte

Helle Varroa

$(\%)$

beschädigte

dunkle Varroa

(\%) signifikant

$r=0,42 ; p=0,00006$

$n=86$;

$1 / y=0,087+0,0076 \bar{x}$
Brutbefall

$r=-0,28, p=0,008$

$n=87$

$y=39,8-0,48 x$

$r=0,47 ; p=0,00001 ;$

$n=83$

$1 / y=0,08+0.0044 x$

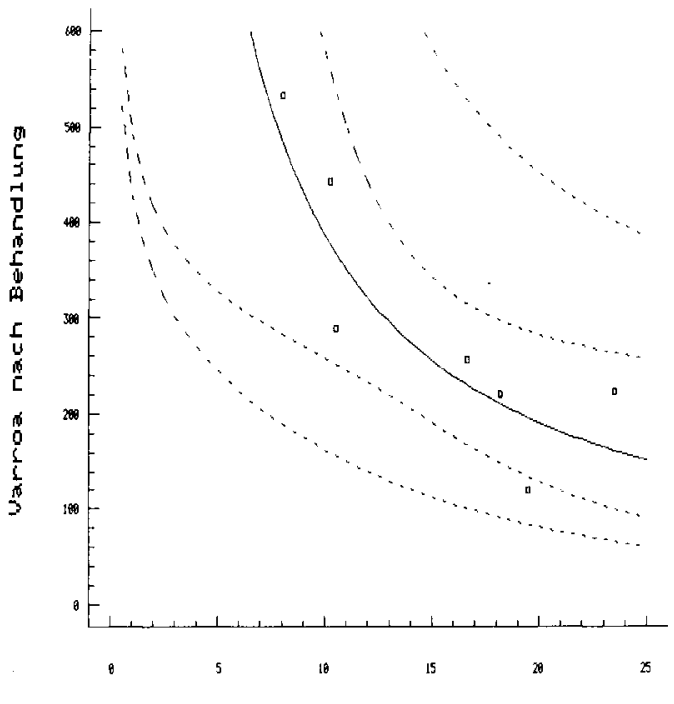

Beschaedigte Varroanilben $(\%)$

Abb 4. Beziehung zwischen dem Prozentsatz beschädigter Varroamilben und dem VarroaAbfall nach einer Behandlung mit Apistan beziehungsweise Bayvarol.

aller 3 Beobachtungsintervalle: 112 untersuchte Milben]; Volk 79: $40 \%$ beschädigte Varroamilben [Summe aller 3 Beobachtungsintervalle: 358 untersuchte Varroamilben]) tatsächlich auf genetische Unter- schiede in diesem Merkmal zurückzuführen sind, müssen weitere Untersuchungen an selektierten Völkern - vor allem auch hinsichtlich der Heritabilität - zeigen.

Wenn ein aktives Abwehrverhalten der Bienen auf das Populationswachstum der Varroa einen Einfluß hat, sollte sich ein solcher auch in der Praxis nachweisen lassen. Tatsächlich weist die gefundene negative Korrelation zwischen dem Varroa-Abfall nach einer chemischen Behandlung mit Apistan bzw. Bayvarol und dem Prozentsatz beschädigter Varroamilben in diese Richtung (Der Varroa-Abfall nach einer einmonatigen Behandlung mit Pyrethroidstreifen entspricht annähernd der Gesamtpopulation an Varroamilben im Bienenvolk).

Als weiteres Indiz kann auch die gesicherte Korrelation'hohe Beschädigungsrate - neidriger Befall (Brut- und Bienenbefall)' gewertet werden.

Sofern der in Abb 4 eingezeichnete Kurvenvereauf auch bei einer größeren Völkerzahl und höheren Befallswerten nur annähernd Gültigkeit hat, sollten allein die von Wallner (1990b) angegebenen Werte von $44-68 \%$ beschädigter Milben - bei selektierten Völkern - ausreichen, die Varroa-Gesamtpopulation unter der Schadensschwelle zu halten. Daraus wird deut- 
lich, welches große Potential einer Varroakontrolle in einem aktiven Abwehrverhalten der Bienen schlummern könnte. Möglicherweise liegt in diesem Auslesekriterium einer der Schlüssel zu einer varroatoleranten Biene.

\section{DANKSAGUNG}

Den Imkern des Instituts für Bienenkunde Gainfarn möchte ich für ihre Hilfe und Ausdauer bei der Probenauswertung herzlich danken. Prof $F$ Ruttner und Imkermeister A Wallner lieferten durch ihre Beobachtungen den Anstoß zu dieser Arbeit und wertvolle Anregungen bei der Durchsicht und Kritik des Manuskriptes.

\section{Summary - Observations on the occur- rence of damaged Varroa mites in natu- ral mite fall of Apis mellifera carnica} colonies. Natural mite mortality reflects the population growth and the reproductive potential of Varroa and also provides information on bees active defence mechanisms. Wallner (1989; 1990a,b; 1991; 1992) was the first to postulate active defence mechanisms based on his observations of mites with damaged legs and cuticle of the idiosoma. These findings were confirmed by Ruttner (1991) and Ruttner and Hänel (1992). In the present study the incidence of damaged mites was recorded in 111 colonies of Apis mellifera carnica at 4 locations in lower Austria from 18th August-18th September 1991. Dead mites were collected via wire-covered inlays in the floor board and counted 3 times during the investigation period. Mites were registered as undamaged or damaged (legs partly or completely amputated, cuticle of idiosoma or cuticle and legs damaged).

The percentage of damaged mites was correlated with bee- and brood infestation level, origin of queens, and the number of Varroa found after treatment with pyreth- roid strips. Details on observed hives have been given in table I. To estimate total mite population, a treatment with Apistan or Bayvarol followed immediately after the last determination of natural mite fall. Data on bee and brood infestation rate were determined once at the beginning of September.

Out of 8452 mites (= total sum of all colonies) 1601 (18.9\%) showed injuries (table II). Light coloured mites (= immature adult females) were injured significantly more frequent $(27.1 \%)$ than dark mites (9.5\%; table II). Figure 1 presents data on injury, which is divided into 9 percentage classes and shows the distribution of colonies according to these particular classes. Only $7.4 \%$ of the colonies (= last 3 classes) had $>30 \%$ damaged mites in their natural mite fall. No significant differences in percentage of damaged mites was observed between the 4 locations or 4 groups of queens from different origins.

Natural mite mortality and the number of damaged mites showed a highly significant linear correlation. These findings are in agreement with those of Ruttner and Hänel (1992). The percentage of natural mite mortality was significantly correlated with bee- and brood infestation rate. A significant correlation was found between percentage of light-coloured damaged mites and brood infestation rate $(\%)$, but not with bee infestation rate (\%). The percentage of dark, damaged mites was significantly correlated both with brood and bee infestation rate (figs 2,3 ). The significant negative correlation between percentage of damaged mites and number of mites found after chemical treatment as well as the negative correlation with bee- and brood infestation rate gives an indication of the influence of active defence behaviour by the bees on Varroa population growth (fig 4). If this behaviour could be increased by breeding and selection measures, it would 
probably constitute an important step towards a Varroa-resistant Carniolan honey bee.

Apis mellifera carnica / Varroa resistance / amputation / defensive behaviour

Résumé - Observations sur la présence de varroas endommagés parmi les acariens morts naturellement dans les colonies d'abeilles Apis mellifera carnica. La mortalité naturelle de l'acarien Varroa jacobsoni reflète la croissance de la population et le potentiel reproducteur du varroa et fournit des informations concernant les mécanismes de défense active des abeilles. Wallner (1989, 1990a,b, 1991, 1992) a été le premier à postuler l'existence de mécanismes de défense active après avoir observé des varroas qui présentaient des pattes et la cuticule de l'idiosome endommagées. Ces faits ont été confirmés par Ruttner (1991) et Ruttner et Hänel (1992). Dans l'étude présente la fréquence des acariens abîmés a été enregistrée dans 111 colonies d'Apis mellifera carnica en 4 endroits de Basse Autriche du 18 août au 18 septembre 1991. Les varroas morts ont été récoltés à l'aide de "langes" recouverts d'une grille et posés sur le plancher de la ruche. Ils ont été comptés à 3 reprises et classés en indemnes ou endommagés (pattes partiellement ou totalement amputées, cuticule de l'idiosome ou cuticule et pattes endommagées). Le pourcentage de varroas endommagés a été mis en relation avec les niveaux d'infestation des abeilles et du couvain, l'origine des reines et le nombre de varroas trouvés après un traitement avec des bandelettes aux pyréthrinoïdes. Le tableau I indique la force des ruches utilisées pour l'expérience. Afin d'estimer la population totale de varroas, un traitement à l'Apistan ou au Bayvarol a été effectué juste après la dernière détermina- tion de la mortalité naturelle. Les taux d'infestation des abeilles et du couvain ont été calculés début septembre.

Sur 8452 varroas (sur l'ensemble des colonies), 1601 (18,9\%) présentent des blessures (tableau II). Les varroas légèrement colorés (femelles adultes immatures) sont plus fréquemment endommagés $(27,1 \%)$ que les foncés $(9,5 \%$, tableau II) et ce de façon significative. La figure 1 , où les blessures sont réparties en 9 classes de pourcentages, montre la répartition des colonies en fonction de ces classes. Seulement $7,4 \%$ des colonies (les 3 dernières classes) ont plus de $30 \%$ de varroas endommagés dans les débris de mortalité naturelle. II n'y a pas de différence significative dans les pourcentages d'acariens endommagés entre les 4 lieux ni entre les 4 groupes de reines provenant d'une origine différente. La mortalité naturelle des acariens et le nombre de varroas endommagés présentent une corrélation linéaire hautement significative. Ces résultats sont en accord avec ceux de Ruttner et Hänel (1992). La mortalité naturelle des varroas est significativement corrélée en pourcentages avec le taux d'infestation des abeilles et celui du couvain. Une corrélation significative a été trouvée entre le pourcentage de varroas clairs et endommagés et le taux d'infestation du couvain, mais pas avec le taux d'infestation des abeilles. Le pourcentage de varroas foncés et endommagés est significativement corrélé avec le taux d'infestation du couvain et celui des abeilles (figs 2 et 3 ). La corrélation significativement négative entre le pourcentage de varroas endommagés et le nombre de varroas trouvés après un traitement chimique, de même que la corrélation négative avec le taux d'infestation du couvain et celui des abeilles est une indication de l'influence du comportement de défense active des abeilles sur la croissance de la population de varroas (fig 4). Si ce 
comportement pouvait être augmenté par la sélection, il pourrait être vraisemblablement une étape importante vers une abeille carnolienne résistance au varroa.

Apis mellifera carnica / résistance à Varroa / amputation / comportement de défense

\section{LITERATUR}

Peng YS, Fang Y, Xu S, Ge L (1987) The resistance mechanism of the Asian honeybee, Apis cerana Fabr, to an ectoparasitic mite Varroa jacobsoni Oud. J Invertebr Pathol 40, 54-60
Ruttner F (1991) Auf dem Weg zu einer varroatoleranten Carnica. Allg Dtsch Imkerz 25, 10 15

Ruttner F, Hänel H (1992) Active defense against Varroa mites in a carniolan strain of honeybees. Apidologie 23, 173-187

Wallner A (1989) Auf der Suche nach der varroaresistenten Biene. Bienenwelt 31, 257-259

Wallner A (1990a) Beobachtungen natürlicher Varroaabwehrreaktionen in meinen Bienenvölkern. Bienenvater 111, 299-300

Wallner A (1990b) Imkern heute. Eigenverlag, Randegg

Wallner A (1991) Varroa-Abwehr. Imkerfreund 46, 4-6

Wallner A (1992) Natürliche Varroaabwehr Volk 13. Bienenvater 113, 57-58, 99-101 